\title{
Introducing safety syringes into a UK dental school - a controlled study
}

\author{
J. M. Zakrzewska, ${ }^{1,2}$ I. Greenwood, ' and J. Jackson, ${ }^{3}$
}

\begin{abstract}
Aim How an appropriate safety syringe was chosen, how the change-over to it was achieved and what outcome measures were used to measure the effectiveness of this change.

Introduction One third of all reported sharps injuries in dental practice are due to the use of non disposable dental syringes with most injuries being sustained during removal and disposal of the disposable needle from the non-disposable syringe.

Method After evaluation of all available disposable safety syringes they were introduced into a dental school after appropriate education of all staff and students. Risk management provided data on all reported needle-stick injuries in the dental school and a control unit using non disposable syringes for a period of two years.

Results Avoidable needle stick injuries reduced from an average of 11.8 to 0 injuries per $1,000,000$ hours worked per year as compared to a control unit who reduced their frequency from 26 to 20 injuries per $1,000,000$ hours worked. The cost of safety syringes is comparable to non-disposable syringes but the reduction in cost of management of needle stick injuries including the psychological effects are significant.

Conclusion Education plays a vitally important role in the effective implementation of the change to safety syringes which is advocated for all dentists.
\end{abstract}

$\mathrm{T}$ he dental metallic cartridge syringe was introduced into dentistry in 1921 and an aspirating plunger was added 36 years later. Since then few modifications have taken place and the dental syringe is such a part of the everyday life of dental practice that all too often it is taken completely for granted. One would hope that there is not a single practice in the United Kingdom that uses nondisposable needles, yet most practices continue to use non-disposable syringes. The use of non-disposable syringes means that needles must be re-sheathed in order for the syringes to be dismantled and the appropriate parts autoclaved. It is during re-sheathing and disposal of the needles that the majority of needle stick injuries occur, especially when done frequently in an environment with trainees and students. ${ }^{1}$ The injuries occur at a time when the syringes are most likely to be contaminated, having been in the patient's mouth. Surveys carried out among US dentists showed

\footnotetext{
1,2 Oral Medicine Department, St. Bartholomew's and The Royal LondonSchool of Medicine and Dentistry, Turner Street, London, E1 2AD ${ }^{2}$ Mortimer Market Centre Dental Clinic, Mortimer Market, London, WC1E 6AU ${ }^{3}$ Dental Auxiliary School St. Bartholomew's and The Royal LondonSchool of Medicine and DentistryTurner Street, London, E1 2AD

${ }^{\star}$ Correspondence to: J. M. Zakrzewska

E-mail: j.m.zak@mds.qmw.ac.uk

REFEREED PAPER

Received 20.10.00; Accepted 05.12.00

(C) British Dental Journal 2001; 190: 88-92
}

that they, on average, sustain 3 injuries a year and $32-33 \%$ of them are related to needles. ${ }^{2}$ In Scotland a survey showed that non-sterile inoculation injuries occurred at a yearly rate of $1.7(\mathrm{SD}=3.2)$ and of these $30 \%$ constituted a moderate or high risk of transmission of infection to the dentist. ${ }^{3}$

It is well known that needle stick injuries can result in transmission of blood borne viruses, including HIV, hepatitis B and C. A survey of occupational injuries in a London teaching hospital in 1994 showed that $7 \%$ of exposures involving blood or body fluids were positive for at least one blood borne virus. ${ }^{4} \mathrm{~A}$ survey in a Californian dental school showed that $17 \%$ of source patients were HIV positive. ${ }^{5}$ Staff who have acquired a needle stick injury from a patient at high risk of, or known to be, HIV positive face at least a three month period of uncertainty about their status which can lead to considerable anxiety and distress. ${ }^{6}$ If following Department of Health recommendations on post exposure prophylaxis they take antiretroviral therapy they are also highly likely to suffer unpleasant toxic side effects which affects compliance. ${ }^{7}$ Although protection against hepatitis $\mathrm{B}$ is available there is, as yet, no vaccine against hepatitis C, whose prevalence in the UK in 1997 was estimated at 2.3 per $1000 .^{8}$ It is, therefore, imperative for all staff to ensure that the risk of acquiring a needle stick injury is reduced to a minimum. It is for this reason that UK hospital infection control policies clearly state 'Handle all sharps carefully, never re-sheath needles, all sharps and needles should be disposed of directly into sharps bins'. The Centre for Disease Control (CDC) guidelines in the US also state that needles should not be recapped but do add that in instances where non disposable syringes are used then a one handed 'scoop' technique or mechanical device should be used. ${ }^{9}$ Until the last few years, there were no suitable disposable dental syringes available to dentists as is borne out by this statement in a textbook on infection control published in 1995 'dentistry has no disposable anesthetic syringe and there is no feasible alternative to recapping. ${ }^{10}$ Manufacturers have been aware of this problem for a long time and there are now several different types of safety syringe on the market. It is, therefore, time for dental schools and practices to reconsider their practice concerning syringes. This involves a major change in any clinical practice and needs to be considered with care. How change can be achieved in clinical practice has become the focus of many articles as it is essential that guidelines and research findings are quickly incorporated into clinical practice. Grol has emphasized in his review on beliefs and evidence in changing practice that several different methods may need to be used in order to effect a change. ${ }^{11}$

It is with all these factors in mind that one dental school comprising some 300 staff (undergraduate, postgraduate students, qualified dentists, dental nurses, hygienists and therapists) decided to make the change over to safety syringes.

This paper aims to describe how an appropriate syringe was chosen, how the change- over to safety syringes was achieved and what outcome measures were used to measure the effectiveness of this change. 
Table I: Comparison of I non disposable and 3 safety syringes to the gold standard

\begin{tabular}{|c|c|c|c|c|c|}
\hline Gold standard & Standard non-disposable & USA Hyposafety & US ultrasafe & Kavo disposable & Safety plus \\
\hline Quickly assembled & No & Yes ++ & Yes ++ & No & Yes ++ \\
\hline Needle attached already & No & Yes & Yes & No & Yes \\
\hline Variety of needles available & Yes & Yes & Yes & Yes & Yes \\
\hline Accept variety of cartridge sizes & Yes & No & No & No & Yes \\
\hline Suitable size for all operators & Mixed types & Very small size & Small size & Small size & Small size \\
\hline Light weight & No & Lightweight ++ & Lightweight ++ & Lightweight + & Depends on handle \\
\hline Variable handles depending on preference & Thumb ring and $\mathrm{T}$ shaped & Thumb ring & Thumb ring & T shaped & $\begin{array}{l}\text { Plastic thumb ring, metal } \\
\text { T-shaped, interligamentary }\end{array}$ \\
\hline Cartridge visible through syringe & Yes & Not clear & Yes & Not clear & Not clear \\
\hline $\begin{array}{l}\text { Wide sheath to protect needle } \\
\text { when syringe not in use }\end{array}$ & No & No & Yes & Yes & Yes \\
\hline Provide effective aspiration & Yes & Yes & Yes & Yes & Yes \\
\hline Able to put in another cartridge if necessary & Yes & Yes & No & Yes & Yes \\
\hline Complete unit disposable & No, syringe left & Yes & Yes & No, syringe left & No, handle left \\
\hline Cartridge disposed with needle & No & Yes & Yes & No & Yes \\
\hline Quick to dispose & No & Yes ++ & Yes++ & No & Yes + \\
\hline Easy to dispose & No & Yes & Yes & No & Yes \\
\hline Autoclaving essential & Yes & No & No & Yes & No \\
\hline Little training required & Need ++ & Little & Little & Need +++ & Need + \\
\hline Cheap & ++ & + & + & + & ++ \\
\hline Risk of injury when used & High & Low & Very low & Low & Very low \\
\hline
\end{tabular}

\section{Method}

The stages used in achieving a change over from non-disposable to disposable (safety) syringes are described below.

1 Collecting the evidence for the need for a change in practice and setting the outcome measures. A dedicated clinic dealing exclusively with patients with blood borne viruses (predominately HIV) had, over a number of years reported several needle stick injuries due to the use of non-disposable syringes. The psychological trauma associated with these injuries was considerable and the clinic members changed to safety syringes as soon as a suitable one was launched on the UK market. A review of the reported incidence of needle stick injuries among staff at a dental school also showed that the dental school was at high risk of sustaining needle stick injuries. The majority of reported needle stick injuries had been sustained during resheathing and dismantling of the syringes or while the needle was lying uncovered in an operating area. These were considered avoidable injuries as they could be prevented by the use of appropriate safety syringes. It was, therefore, clear that the main outcome measure to be used to assess the value of the change over to safety syringes would be that of reduction in the number of avoidable needle stick injuries attributable to the use of the syringes. It would not be possible to reduce those injuries sustained due to patient or operator unexpected movements. Data on injuries for the previous 3 years and then for 2 years after introduction of the safety syringes was collected from risk management. Apart from the investigators, staff were not aware that this monitoring was being carried out. Staff numbers for each year were collected and an estimate made of the number of hours worked. Secondary outcome data on costs of the syringe and the management of needle stick injuries were obtained from Central Sterile Supplies Department (CSSD), Supplies Department and Occupational Medicine.

2 Testing of the product. Two qualified dentists in the dedicated clinic contacted manufacturers of syringes and eventually found four different types of safety syringes. They evaluated them on trial for a month, although two models used $1.8 \mathrm{ml}$ cartridges rather than the standard UK size of $2.2 \mathrm{ml}$ and so were not considered of use in the UK. In their evaluation they used the basic criteria as laid down by the American Dental Association Council on Dental Materials ${ }^{12}$ and others as described by Malamed. ${ }^{13}$ The results of the evaluations are shown in table 1 . The four syringes are pictured in figure 1 and clearly illustrate the component parts that are disposable and those that need to be autoclaved. The handle of the Septodont Safety Plus syringe does not require autoclaving unless it has been contaminated with blood and saliva but it should be disinfected by immersal in hypochlorite solution of appropriate strength and for sufficient time as used for other dental items such as shade guides. Details on how to assemble the Ultrasafe and Septodont Safety plus systems can be found in Malamed's Handbook on Local Anesthesia. ${ }^{13}$ Further talks with manufacturers resulted in improved designs and finally one product was chosen and adopted as the syringe of choice in the dedicated clinic.

3 Choosing a moment for introducing the change within the dental school. Due to major changes in the way that instruments were to be sterilized in the future a dental school was having to order extra equipment to cope with the increased turnover time of instruments. Clinicians were being asked to choose a new syringe and this was used as a moment to introduce the new safety syringes as money had been identified for the purchase of new syringes.

4 Convincing key players of the need for change. One of the authors who worked at the dedicated clinic also worked at a UK dental school and had been convinced of the success of the new safety syringes in the dedicated clinic. She was prepared to introduce them into the teaching hospital. The data on needle stick injuries, samples of the product and cost analysis were all used to introduce the concept of a safety syringe to key clinicians in the restorative dentistry department, service managers, clinical directors and nursing staff.

5 Ensuring adequate supplies and means of disposal. Negotiations were entered into with the chosen supplier in order to ensure that adequate supplies would be available to allow the whole school to change at the same time. In view of the increased bulk of the safety syringes new waste disposal bins had to be ordered and distributed round the clinics. On the other hand waste paper used by CSSD to wrap up the non disposable syringes was reduced. 
Table 2 Number of sharps injuries sustained in the dental school and a control unit 3 years prior (years I-3) and 2 years (years 4-5) post introduction of safety syringes

\begin{tabular}{|c|c|c|c|c|c|c|c|c|c|c|}
\hline Injury & $\begin{array}{c}\text { Year I } \\
\text { Qualified }\end{array}$ & $\begin{array}{l}\text { Year I } \\
\text { Trainee }\end{array}$ & $\begin{array}{c}\text { Year } 2 \\
\text { Qualified }\end{array}$ & $\begin{array}{c}\text { Year2 } \\
\text { Trainee }\end{array}$ & $\begin{array}{c}\text { Year } 3 \\
\text { Qualified }\end{array}$ & $\begin{array}{l}\text { Year3 } \\
\text { Trainee }\end{array}$ & $\begin{array}{c}\text { Year4 } \\
\text { Qualified }\end{array}$ & $\begin{array}{c}\text { Year } 4 \\
\text { Trainee }\end{array}$ & $\begin{array}{c}\text { Year } 5 \\
\text { Qualified }\end{array}$ & $\begin{array}{c}\text { Year } 5 \\
\text { Trainee }\end{array}$ \\
\hline \multicolumn{11}{|l|}{ School using safety syringe } \\
\hline Number of staff at risk & 68 & 173 & 68 & 170 & 68 & 186 & 68 & 176 & 68 & 176 \\
\hline Total number of sharps injuries & 5 & II & 5 & 8 & 4 & 9 & 4 & 3 & 4 & I \\
\hline Number relating to syringes & 2 & 5 & I & 4 & 2 & 4 & 2 & 2 & 0 & 0 \\
\hline Number avoidable injuries & I & 5 & I & 3 & 2 & 3 & 2 & I & 0 & 0 \\
\hline Number of staff at risk & 39 & 20 & 39 & 20 & 39 & 20 & 39 & 20 & 39 & 20 \\
\hline Total number of sharps injuries & 4 & 3 & 3 & I & 2 & 2 & 4 & I & 2 & 0 \\
\hline Number relating to syringes & 3 & I & 2 & 1 & I & 2 & 4 & 0 & 2 & 0 \\
\hline Number avoidable injuries & 2 & I & l & i & i & 2 & $2^{*}$ & 0 & $I+I^{*}$ & 0 \\
\hline
\end{tabular}

Trainee $=$ dental student, student dental nurse or student therapist/hygienist

Avoidable $=$ any injury that relates to removal and disposal of needle or its protection when not in use that would not occur when using the ideal safety syringe,

* near misses reported by CSSD.ie: unsheathed needles on syringes.

It is assumed that staff work a 37.5 hour week and work for 46 weeks a year. The estimated number of probable syringe contacts per week in the school is 1640 whereas for the unit using the metal syringes it is 270 .

Storage areas for the syringes were also identified.

6 Setting up a clear protocol for changeover. All key staff involved in the change over were aware of the need for high attendance at training sessions and the date of introduction of the new syringes which was widely publicised.

7 Training of staff. Over a period of two weeks multiple training sessions were organised with the manufacturers' personnel coming in to ensure that the technique was carefully explained, demonstrated and tested. All grades of staff were included as well as all the dental students and trainee nurses. Dental students were not allowed to use the new system until they had been signed up as attending the sessions.

8 Ensuring smooth change over after training. Once the staff had undergone training the new syringes were introduced throughout the dental school. The CSSD department was alerted to the need to sterilise the handles and to replace them after a hundred cycles. Representatives from the manufacturers then attended at regular intervals to pick up on any problems and to institute changes if possible. Training videos were made and given to the school to ensure further training of new staff after a temporary new member of staff sustained an injury due to lack of training. All new junior dental staff are introduced to the new syringes during their induction period.

9 Follow up. Careful monitoring has continued and all staff are encouraged to highlight any problems that are encountered. Close contact is maintained with the manufactures so that any modifications can be made if necessary. Preliminary use of the syringes, especially among dental students, showed that the internal diameter of the needle was too small as increased pressure on the handle resulted in bending of the needle. Consequently the needles were re-designed and their internal diameter enlarged without any outside increase in diameter. Less pressure is now applied while injecting resulting in fewer bent needles, more flexibility and decreased discomfort to the patient. Dental students knowledge is tested formally during their in-course assessment when they are observed preparing a syringe for use and then disposing of it.

A busy surgical unit was used as a control. Although they had undergone training they considered that there was insufficient evidence to prove that the injuries occurring during disposal could be reduced by the use of safety syringes. Increased awareness of safety issues was considered sufficient.

\section{Results}

Of the four safety syringes the Septodont Safety Plus system was chosen as it fulfilled most of the requirements and this is illustrated in figure 1 , syringe 1 .

Table 2 shows that the majority of sharps injuries in the dental school are due to the use of syringes and that most could have been avoided with the use of a safety syringe. More injuries are sustained by trainees and students than qualified staff. The main outcome measure showed that a reduction in avoidable needle stick injuries had been achieved. In the dental school the frequency of avoidable needle stick injuries fell from a pre-change average of 11.8 to 0 per $1,000,000$ hours worked per year in the second year of use of the safety syringes. Some reduction was noted in the surgical unit as shown in figure 2 but this was not as marked and was the same over the two years. Incidence of avoidable needle stick injuries per 1,000

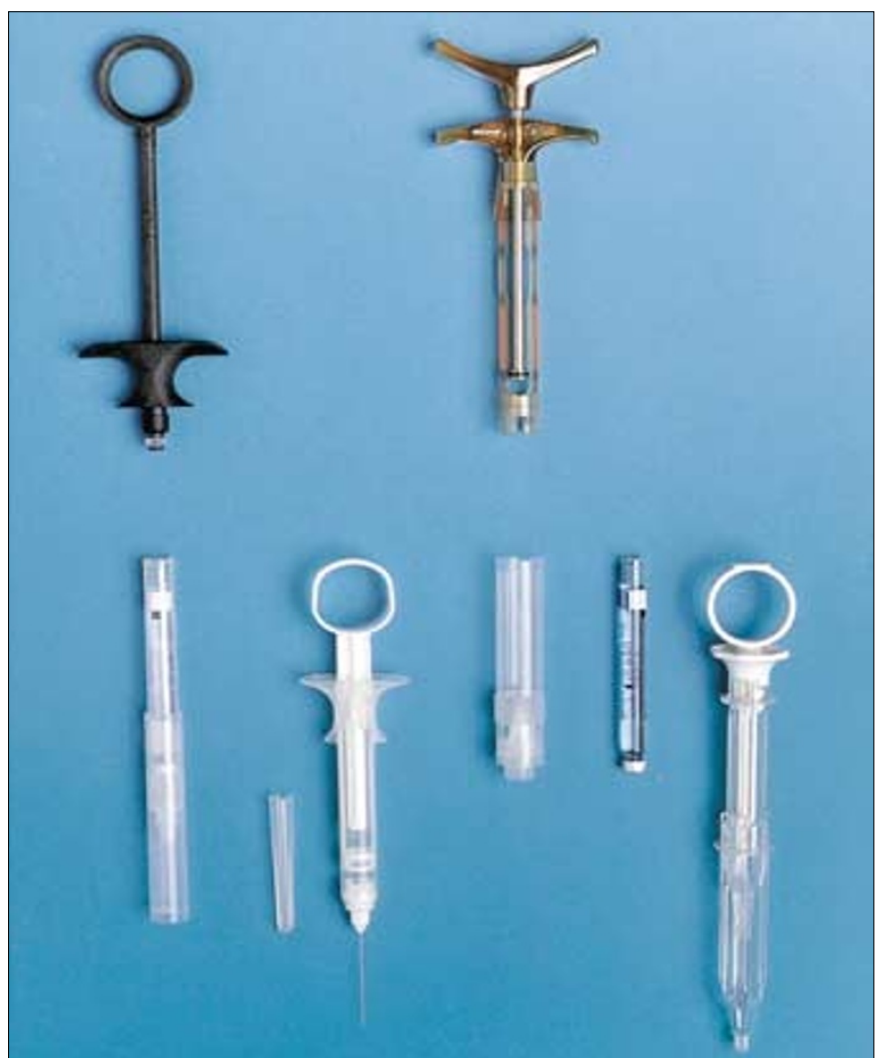

Fig. I Examples of the four safety syringes currently on the market. Number one is Safety plus, number 2 is USA hyposafety, number 3 is Kavo disposable and number 4 is USA ultrasafe. Their characteristics are discussed in table I. All items below the line are disposable. 
employees also fell in the dental school, from an average of 20.5 to 0 in the second year, whereas in the surgical unit it remained the same - an average of 45.2 per 1,000 employees in the three years prior to introduction to 33.9 per 1,000 in the two years after the introduction. Due to the small numbers of injuries the change-over has not resulted in a statistically significant change but there is a definite trend. In the dental school there is an estimated 1640 needle contacts a week whereas in the surgical unit it is 270 per week.

Post introduction of the safety syringes four injuries were sustained in the dental school in the first six months. Three of these were avoidable and had been recorded on the accident form to be related to lack of training. The personnel involved were a temporary nurse, a trainee nurse and a qualified dental nurse. The other injury occurred due to movement of the patient during an injection. In the surgical unit still using metal non disposable syringes two injuries were due to patient movement. The other reports were of 'near misses' in which 3 workers in CSSD found used unsheathed needles still attached to the dental syringes when being returned for autoclaving. The needles had been bent so badly that they would have been impossible to re-sheath using the narrow sheaths available with those needles.

The current cost of a 100 safety needles and syringes and one handle in the dental school is $\mathfrak{E} 5.95$, extra handles cost $\mathfrak{E} 1.25$ each. Needles used for non disposable syringes cost $\mathfrak{E} 6.06$ per hundred and a non-disposable syringe costs $£ 15$ and needs replacing every two years. Sterilization costs $20 \mathrm{p}$ per item. The cost of a safety syringe is therefore around $18 \mathrm{p}$ whereas a non disposable syringe costs around $25.8 \mathrm{p}$.

The following estimates on managing a recipient such as an $\mathrm{E}$ grade nurse were based on the cost of managing needle stick injuries in cases were the source patient is HIV negative (requires no intervention) or HIV positive (when staff may or may not elect for post exposure prophylaxis). The costs include administrative and staff costs, 30 minute consultation and blood tests.

- A needle stick injury, no drugs and no starter pack:

- A needle stick injury, starter pack no further drug course:

$\mathfrak{£} 136.04$

- A needle stick injury, full drugs course, no sickness absence:

$\mathfrak{E} 296.89$

E2,151.70

- A needle stick starter pack, full drugs course, sickness absence:

- Extra three one hour counselling sessions if requested:

In the data obtained from risk management one of the source patients was HIV positive but due to confidentiality it was not possible to ascertain whether the recipient took post exposure prophylaxis. In the Trust to which the dental school belongs it was estimated that 1 in 119 incidents are reported to the Communicable Diseases Surveillance Centre. Under-reporting is common but difficult to estimate as recipients have a variety of routes by which to seek support and maintenance of confidentiality is a high priority in order to encourage reporting.

Close contact with the manufacturers has led to continual improvement in the design of the safety syringe which has improved their acceptance among the dental staff. The barrels are now tighter and the wider bore needles are a marked improvement. Needles are more flexible and less likely to become blocked and do not appear to result in breakage given their thinner walls. Work is continuing to make the plastic clearer so that it is easier to detect blood when aspirating and to improve aspiration. It has been found that on average it takes staff one month to completely familiarize themselves with the new system. Although the syringes take just as long to put together, their disposal is markedly faster and safer. Continued education is essential and a dental infection control nurse has been designated to ensuring that all new staff including students are trained in the correct use of the safety syringes.

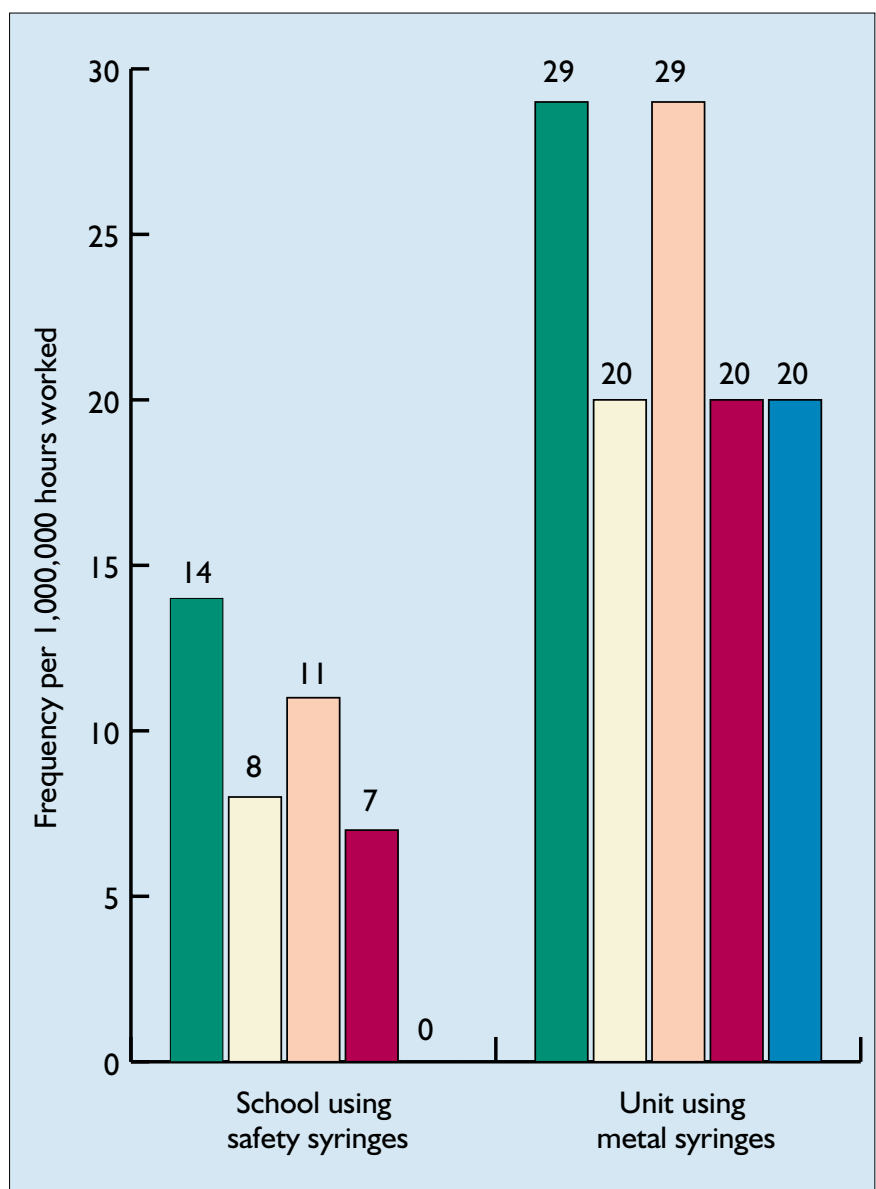

Fig. 2 Frequency of reported avoidable needle stick injuries three years prior to the use of safety syringes and 2 years after their introduction in a dental school. Frequency is defined as the frequency per I,000,000 hours worked based on a 37.5 hour week and 46 week year.

\section{Discussion}

It is possible for a dental school to change to safety syringes provided key issues are addressed and it does result in a reduction in the number of avoidable needle stick injuries.

It was acknowledged from the outset that effecting a change to a lighter, plastic, safety syringe was nearly equivalent to the change of routine wearing of gloves during dental procedures. The benefits of glove wearing are now well recognised and we have shown that a reduction in number of avoidable needle stick injuries can be achieved by changing to safety syringes provided sufficient training has been given. The benefit may not be as marked in general dental practice where the number of avoidable needle stick injuries may be smaller.

It needs to be remembered that underreporting of needle stick injuries is commonplace and has been reported to be as high as $26 \%$ in a California Teaching Hospital. ${ }^{5}$ The number of reported injuries is relatively small but these are some of the highest figures for a clinical directorate in this Trust and show that the majority of sharps injuries occur while dismantling the syringes.

The reduction in injuries could have been due to raised staff awareness as a result of the intense training and highlighting of needle stick injuries and not due to the new safety syringes as shown by the reduction in year 2 prior to introduction and by the surgical units figures. However analysis of the timing of the injuries after introduction of safety syringes shows that all the injuries reported after the change-over occurred in the first six months of the change over and in the last 18 months no injuries were reported. The avoidable needle stick rate is reduced and sustained. This would support 
the conclusion that the new safety syringes contributed significantly to these reductions but that increased awareness is also important.

This study in common with others showed that it is the unqualified staff that are at highest risk of injury. ${ }^{5}$ This is of special importance in dental schools where staff are training and are therefore more vulnerable to injuries. ${ }^{1}$ Education needs to be continued as Gyawali et al. have stressed in their review of injuries. ${ }^{4}$ This is especially important currently, as new staff joining the school may not have used this system in the previous workplace. One of our reported injuries was sustained by a temporary member of staff. To ensure that all dental students attend training sessions they are formally tested on their ability to use the new syringes during their in-course assessments. No dental student has sustained an avoidable needle stick injury since the introduction of the safety syringe. New dental students are now being taught how to use the product within the first few weeks of starting their dental course and none have sustained any injury whilst practising which was commonplace previously. Staff did find that it took some time to get used to the new syringes and it is important to acknowledge this in order to stop them going back to their old practices. Malamed also acknowledges the ease with which these systems are learned and of the importance of using them. He states that 'a safety syringe system is strongly recommended.' ${ }^{\prime 3}$

In our study the dental nurse trainees were most frequently involved in needle stick injuries. This was surprising given the local policy that the dentist is responsible for clearing the area of all sharps before handing over to the nurses. This study has highlighted this deficient practice and it is an area of education that is now being addressed.

It has also been argued that when dental students move out into general dental practice they will be unfamiliar with non-disposable syringes and could, therefore, be at increased risk of sustaining needle stick injuries. It is the contention of the authors that dental students, once qualified, and joining a practice should feel sufficiently confident with the use of safety syringes that they will ask their colleagues to change to the new system rather than adapt to outdated practices. The need to effect a change over to safety syringes and to increase awareness of needle stick injuries is further highlighted by a recent report from the Dental Protection Society which quotes the case of a nurse who sustained a needle stick injury while attempting to re-sheath a needle. ${ }^{14}$ It was noted that she had neither received instruction about infection control nor had she been provided with any type of re-sheathing device. The nurse was successful in her claim alleging severe anxiety and depression following the needle stick injury. Employers could be liable if their employees attempt to sue them for exposing them to products that have a higher risk for needle stick injury when safer equipment is now readily available. The State of California has introduced legislation that requires all health care employers to provide sharps safety devices which in the field of dentistry is interpreted as a 'safety needle with engineered built-in safety mechanisms. ${ }^{15}$ Other States have introduced bills with similar regulations. Concern, however, has been expressed that the safety and efficacy of the current devices has not been verified. This study provides evidence that a change to safety needles does result in a substantial change in the number of needle stick injuries in a dental school especially if combined with increased awareness.

The price of the new safety syringes has fallen considerably and there is little cost difference between the two systems. The cost of a needle stick injury is not insignificant even if no treatment is required. Not only are there physical costs but the psychological effects are considerable. ${ }^{6}$ Two of the authors have had considerable experience in dealing with the short and long term effects that staff suffer after sustaining needle stick injuries from patients with HIV and these must not be underestimated. Any reduction in needle stick is therefore psychologically significant. Counselling is often needed for recipients of injuries even if the source patient was not at risk of a blood borne virus. It must also be remembered that some staff do not have an adequate response to Hepatitis B vaccination and that no vaccination is available for Hepatitis C. These two conditions also cause considerable morbidity and mortality.

Working closely with the manufacturers will enable changes to the design to be introduced so improving the product even further. Occupational blood exposures have been steadily falling and this figure can be further improved by the introduction of safety syringes not only into dental schools but also in the community dental services and general dental practice. ${ }^{16}$

We are grateful to: the risk management team at Barts and the Royal London NHS Trust who provided the relevant data, Dr D.Samarawickrama for first introducing the new syringes into his department, Dr E. Davenport for organising the student assessment, DrD. D'Auria for cost data and help with analysis, Ms Turner for statistical advice and all the dental healthcare workers who took part in the study. We are indebted to Septodont for their supplies, training and help.

\section{Appendix}

Manufacturers of syringes discussed in table 3 and illustrated in figure I

I Ultrasafe Safety Syringes Inc 250 W. Coloroado Blvd. Suite 101 Arcadia CA 91007

2 Hyposafety cartridge Syringe MPL Technologies Inc A SoloPak Company Franklin Park, IL 60131-2116

3 Kavo disposable syringe Kavo Dental Ltd 77 Gloucester Rd Patchway Bristol BS34 5JQ

4 Safety Plus Septodont Deproco UK Ltd, Units R and S, Orchard Business Centre, St Barnabas Close, Allington, Maidstone, Kent, MEI6 OJZ

1 McDonald R I, Walsh L J, Savage N W. Analysis of workplace injuries in a dental school environment. Aust.Dent.J. 1997;42:109-113.

2 Siew C, Gruninger S E, Miaw C L, Neidle E A. Percutaneous injuries in practicing dentists. A prospective study using a 20-day diary. J Am Dent. Assoc 1995;126:1227-1234.

3 Gore S M, Felix D H, Bird A G, Wray D. Occupational risk and precautions related to HIV infection among dentists in the Lothian region of Scotland. J Infect 1994;28:209-222.

4 Gyawali P, Rice P S, Tilzey A J. Exposure to blood borne viruses and the hepatitis B vaccination status among healthcare workers in inner London. Occup Environ Med 1998;55 :570-572.

5 Ramos-Gomez F, Ellison J, Greenspan D, Bird W, Lowe S, Gerberding J L. Accidental exposures to blood and body fluids among health care workers in dental teaching clinics: a prospective study. J Am Dent.Assoc 1997;128:1253-1261.

6 David H T, David Y M. Living with needlestick injuries. J Can Dent Assoc 1997;63:283-286.

7 Parkin J M, Murphy M, Anderson J, El-Gadi S, Forster G, Pinching A J. Tolerability and side effects of post-exposure prophylaxis for HIV infection. Lancet 2000;355:722-723.

8 Neal K R, Dornan J, Irving W L. Prevalence of hepatitis C antibodies among healthcare workers of two teaching hospitals. Who is at risk? BMJ 1997;314:179-180.

9 Anonymous. Recommended infection-control practices for dentistry, 1993. Centers for Disease Control and Prevention. MMWR Morb Mortal Wkly Rep. 1993;42:1-12.

10 Cottone J A, Terezhalmy G T, Molinani J A. Practical Infection Control in Dentistry. USA: Williams and Wilkins, 1995:

11 Grol R. Personal paper. Beliefs and evidence in changing clinical practice. BMJ 1997;315:418-421.

12 Council on Dental Materials and Devices. New American National Standards Institute/American Dental Association Specification No 34 for Dental Aspirating Syringes. J Am Dent Assoc 1978;236-238.

13 Malamed S F. Handbook of local anesthesia. USA: Mosby, 1997:

14 Anonymous. Dental Protection Annual Report. Building confidence in dental practice. Medical Protection Society. 13. 1999. London.

15 Cuny E J, Fredekind R, Budenz A W. Safety needles. New requirements of the Occupational Safety and Health Administration bloodborne pathogens rule. J Calif Dent Assoc 1999;26:525-530.

16 Cleveland J L, Gooch B F, Lockwood S A. Occupational blood exposures in dentistry: a decade in review. Infect Control Hosp Epidemiol 1997;18:717-721. 